\title{
Characteristics of the expression of KAI1/CD82 and PDGFR $\beta$ and their impact on glioma progression
}

\author{
Michal Paradowski ${ }^{1}$, Malgorzata Bilinska ${ }^{1}$, Julia Bar ${ }^{2}$ \\ ${ }^{1}$ Department of Neurology, Wroclaw Medical University, ${ }^{2}$ Department of Immunopathology and Molecular Biology, Wroclaw Medical \\ University, Wroclaw, Poland
}

\begin{abstract}
The biological features of glioma cells may define their clinical outcome. Little is known about the interactions between KA/1/CD82 metastatic suppressor protein and PDGFR $\beta$ in gliomas. The aim of the study was to examine $K A / 1 / C D 82$ and PDGFR $\beta$ expression in gliomas in order to find the impact of these proteins on progression of the tumors. PDGFR $\beta, K A / 1 / C D 82$ protein expression and mRNA of genes were evaluated on eighty four paraffin-embedded tissue of gliomas using immunohistochemical staining and RT-PCR analysis. The PDGFR $\beta$ expression was higher in IV/III than in I/II glioma grades $(p=0.0004)$. The level of MRNA PDGFR $\beta$ was associated with the degree of PDGFR $\beta$ immunoreactivity. Downregulation of KA/1/CD82 was associated with tumor malignancy $(p=0.007)$. The increased level of KA/1/CD82 gene expression (3-4-fold) was found in gliomas with strong KA/1/CD82 immunoreactivity. The parallel KA/1/CD82 and PDGFR $\beta$ expression was more significantly associated with cases in a group graded as III and IV than in a group graded as I/II $(p=0.002)$.

We found that a loss of KAII/CD82 and an increase in PDGFR $\beta$ expression in gliomas relate to a progressive tumor growth. The correlation between PDGFR $\beta$ and KAI1 expression in high grade gliomas suggests that a direct or indirect interaction between these proteins might have an impact on cell motility and invasive behavior of the tumor.
\end{abstract}

Key words: gliomas, KAl1/CD82, PDGFRß, immunohistochemistry, RT-PCR.

\section{Introduction}

Gliomas are the most frequent primary neoplasms of the central nervous system (CNS). Most of them are characterized by their extensive invasion into the brain parenchyma $[17,19,20]$. Grade $1 /$ II astrocytomas are slow-growing tumors without aggressive features, whereas grade III and IV gliomas possess a malignant phenotype associated with high proliferative activity and vascular formation $[18,19]$. Glioblastoma (grade IV) is one of the most aggressive and deadly malignant brain tumors with an average survival time of 15 months after diagnosis $[8,13,18,20]$. Most primary glioblastomas develop de novo but some parts of diffuse astrocytomas grade II and III may progress to grade IV as secondary glioblastomas $[12,25]$.

The high infiltration capacity of individual glioma cells is related to the unique biological features of these cells $[5,12,18]$. According to some authors, the migratory behavior of glioma cells observed during the tumor progression might be a result of the activa- 
tion of surface receptors and signaling pathways [19]. A number of alternations in different genes have been identified in human gliomas and some of them are involved in glioma progression [8]. Among the growth factors, the platelet-derived growth factor (PDGF) has been well described in glioblastomas $[19,20]$.

A lot of data suggested that the PDGF receptor activates a number of downstream signal transduction pathways including PI3K/Akt/mTOR and Ras/ Raf/MAPK pathways and may play an important role in both normal development and tumorigenesis of the CNS $[19,20]$. Overexpression of PDGF ligands and receptors are frequent events in human gliomas regardless of the tumor grade. Their expression pattern in gliomas suggests the presence of autocrine and paracrine stimulatory loops [20]. It was revealed that PDGF signaling alone may be sufficient to induce glioma formation [10]. Blocking of PDGF signaling strongly implies that PDGF signaling is necessary and sufficient to maintain the malignant phenotype in the in vitro model [31].

The specific biological mechanisms that mediate tumors' invasive nature are still unknown [30]. Glioma progression may be compared to other solid tumor metastasis process. This process involves a molecular mechanism which requires the contribution of a multiple gene alteration $[15,30]$. The role of metastasis suppressor genes has been discussed very rarely in gliomas $[8,19]$. The most interesting is the KAl1/CD82 gene originally identified as a putative metastasis suppressor gene for prostate cancer [9]. The KAl1/CD82 gene is a member of the tetraspan transmembrane super family (TM4SF) [30]. KAl1/CD82 is expressed in various human tissues and plays an important role in cell fusion, adhesion, migration, signaling, fertilization, differentiation and invasion [11]. Downregulation of both KAI1/CD82 mRNA and protein expression is observed during progression and increased invasive behavior of tumors $[7,15,30]$. Several mechanisms have been proposed by which KAI1/CD82 might influence and control tumor cell behavior [29]. It was established that KAI1/CD82 plays an important role in regulating melanoma cell migration through the controlling of Rho and GTPases signaling activity [23]. Another study revealed that KAI1/CD82 might have an inhibitory role in the PI3K/AKT pathway $[1,6,23]$. So far, the correlation between KAI1/CD82 expression and the signal transduction pathway has been examined in breast and ovarian cancers [29].
Little is known about the interaction between the members of metastasis suppressor genes and kinase receptors in primary brain tumors and their role in glioma cell behavior. The relationship between KAI1/CD82 and tyrosine kinase receptor PDGFR $\beta$ has not been evaluated in gliomas. To investigate the possible suppressive role of KAI1/CD82 in gliomas and their influence on PDGFR $\beta$ expression we examined KAI1/CD82 and PDGFRß expression and the relation between them in gliomas in order to find the impact of these proteins on progression of gliomas.

\section{Material and methods}

The study was performed on tissue sections from 84 patients diagnosed with primary gliomas hospitalized in the Clinic of Neurosurgery of the Wroclaw Medical University, Poland between 2007 and 2012. Tumor tissues were obtained at initial surgery. None of the patients received any treatment before the operation. All tumors were histologically verified to confirm the diagnosis, histological type and tumor grade according to established criteria classification of the central nervous system tumors by the World Health Organization (WHO) [17]. Based on the WHO classification, gliomas were subdivided into the following groups: grade I -6 cases ( 6 cases of pilocytic astrocytoma), grade $I I-24$ cases (22 cases of fibrillary astrocytoma, 2 cases of oligodendroglioma), grade III - 15 cases (12 cases of anaplastic astrocytoma, 3 cases of anaplastic oligodendroglioma) and grade IV - 39 cases of glioblastoma multiforme.

The study was conducted in accordance with the declaration of Helsinki. This study was approved by the Local Ethic Committee of Human Research of the Medical University of Wroclaw, Poland (permission no. 37/2012). Written informed consent was obtained from all participants.

\section{Immunohistochemical staining}

Immunohistochemical staining (IHC) for the analyzed proteins was performed on paraffin-embedded tissue using the Universal DakoCytomation LSAB + Kit, Peroxidase procedure (LSAB+ Kit:HRP, Dako, Copenhagen, Denmark) and the following primary monoclonal antibodies: anti-KAl1 (G2) (Santa Cruz Biotechnology, USA) and anti PDGFR $\beta$ (28E1) (Cell Signaling Technology, USA).

Five-micrometer sections from one selected block from each lesion were deparaffinized and boiled for 
$3 \times 5$ minutes for each antibody in citrate buffer $(\mathrm{pH} 6.0)$ at $700 \mathrm{~W}$ in a microwave oven. After the microwave treatment, the tissue sections were slowly cooled for 20 minutes. Endogenous peroxidase reactivity was blocked with $3 \% \mathrm{H}_{2} \mathrm{O}_{2}$ and nonspecific tissue reactions with $10 \%$ BSA (bovine serum albumin). Tissue specimens were incubated with primary antibodies (anti-KAl1 anti-PDGFR $\beta$ ) overnight at $4^{\circ} \mathrm{C}$. Following washing with $0.1 \mathrm{M}$ Tris-buffer, $\mathrm{pH}=7.4$ (TBS), the tissue specimens were incubated with a secondary biotinylated rabbit antibody, anti-mouse IgG (Dako, Copenhagen, Denmark) and with streptavidin-horseradish peroxidase-conjugated (Dako) both for 15 minutes at room temperature. After washing with TBS, the antigen-antibody reaction was visualized by DAB (3,3'-diaminobenzidine) (Dako, Denmark) as a chromogen (8 minutes, room temperature). Sections were counterstained with hematoxylin and mounted. The incubation buffer (TBS) without the primary antibody was used as a negative control. The internal positive controls were performed according to the manufacturer's protocol.

The preparations were evaluated under a BX-51 Olympus light microscope. The localizations, distributions and intensity of immunostaining were evaluated in the tissue sections. For KAI 1 and PDGFR $\beta$ membrane immunostaining was considered as positive when at least $10 \%$ of tumor cells were stained.

The intensity of staining was scored as 0 for negative, + weak, ++ moderate, and +++ strong. The immunohistochemical analyses were interpreted without prior knowledge of the clinical information.

\section{Reverse transcription polymerase chain reaction}

For reverse transcription polymerase chain reaction (RT-PCR) analysis of KAI1/CD82 and PDGFR $\beta$ genes, immunopositive cases for both proteins were divided into three groups: $10-40 \%$ positive tissue, $41-70 \%$ and $71-100 \%$ positive tissue. Each group consisted of 10 cases.

We used the method of RT-PCR for analysis of KAI1/CD82 and PDGFR gene expression. RNA was isolated from the freezing tissue of 30 patients with gliomas (which revealed a different level of KAI1/CD82 and PDGFR $\beta$ expression in IHC staining) according to the method of RNeasy Plus Mini from Qiagen. The reverse transcription was performed with QuantiTect Reverse Transcription kit (Qiagen). The estimation of KAI1/CD82 and PDGFR $\beta$ gene expression was performed in Rotor-Gene TM. The reaction mixture for RT-PCR volume of $25 \mu \mathrm{l}$ contained $2 \mu \mathrm{l}$ complementary DNA (CDNA) and $2 \mu$ gene sequences of primers for KAl1/CD82 (Hs CD82 1 SG Quant Tect Primer Assay Qiagen), PDGFR $\beta$ (Forward - 5'-AATGTCTCCAGCACCTTCGT-3'489-509, Reverse - 3'-AGCGGATGTGGTAAGGCAATA-5')(1177-1156)andreagents Rotor-Gene SYBR Green Master Mix and RNase-free water. The standard curve plotted on the basis of the reference gene peptidyl prolyl isomerase C (cyclophilin C, CYCC, Hs_PPIA Quant Tect Primer Assay, Qiagen) with a different concentration; diluted 10-, 100- and 1000 -fold. The RT-PCR reaction consisted of one cycle PCR initial activation step of $95^{\circ} \mathrm{C}$ for 5 seconds, followed by 40 cycles of $95^{\circ} \mathrm{C}$ for 5 seconds and $60^{\circ} \mathrm{C}$ of 10 seconds.

\section{Statistical analysis}

Correlations between KAI1 and PDGFR $\beta$ expression and glioma grade malignancy were statistically studied by $\chi^{2}$ test. Associations between KAI 1 and PDGFR $\beta$ expression were analyzed by Spearman's rank correlation. Differences were considered as significant when $p \leq 0.05$.

\section{Results \\ PDGFR $\beta$ expression and analysis of mRNA PDGFR $\beta$ levels in gliomas}

Membrane PDGFR $\beta$ expression was found in $44 / 84(52.3 \%)$ gliomas. The majority of cases revealed PDGFR $\beta$ immunopositivity in 10-40\% of tumor tissue. In the vast majority of gliomas, the immunoreactivity for PDGFR $\beta$ was observed in the group of cells distributed in different parts of tumor tissue (Fig. 1). The strong reactivity for PDGFR $\beta$ (70-100\% positive tissue) was found only in $9.09 \%$ of cases. In glioblastomas PDGFR $\beta$ expression was stronger than in fibrillary astrocytomas and oligodendrogliomas ( $p=0.0001)$. The PDGFR $\beta$ expression revealed differences in IV/III tumor grades versus I/II glioma grades $(p=0.0004)$ (Fig. 2). Similar differences were found for the mean value for PDGFR $\beta$ expression between low grade gliomas (I and II) [Gl $1.666 \pm 4.082$ (SD), Gll $6.25 \pm 19.067$ (SD)] compared with high grade gliomas (III and IV) [GIII $20.666 \pm 22.834$ (SD), GIV $31.794 \pm 28.457$ (SD)], $p=0.001$. In gliomas showing PDGFR $\beta$ immunoreactivity of $70-100 \%$ of tumor tissue, the level of mRNA PDGFR $\beta$ was higher (increased 3-5-fold) compared to 


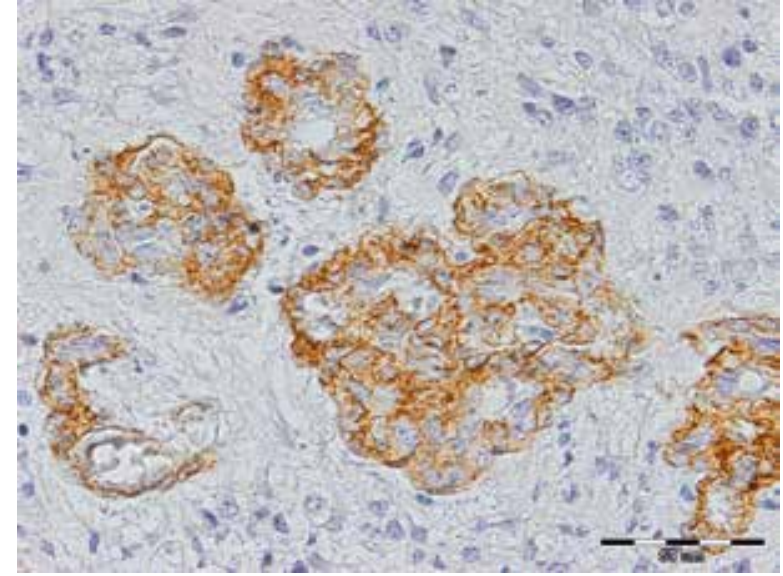

Fig. 1. In glioblastoma tissue, PDGFR $\beta$ is strongly expressed in cell membrane (avidin-biotin staining $\times$ 200).

cases with PDGFR $\beta$ immunoreactivity of $40-70 \%$ of positive cases (increased 1.5-2 fold).

\section{KAI1/CD82 expression and analysis of mRNA KAI1/CD82 levels in gliomas}

KAI1/CD82 immunostaining was observed on the membrane and in the cytoplasm of tumor cells in $75.0 \%$ of gliomas. The membrane staining dominated and was found in a different range of tumor cells (10-100\% tumor tissue) in individual gliomas. Only $17.4 \%$ of cases showed strong immunoreactivity for KAI $1 / C D 82$ ranging between $70 \%$ and $100 \%$ positive tissue (Fig. 3). Statistical differences were observed

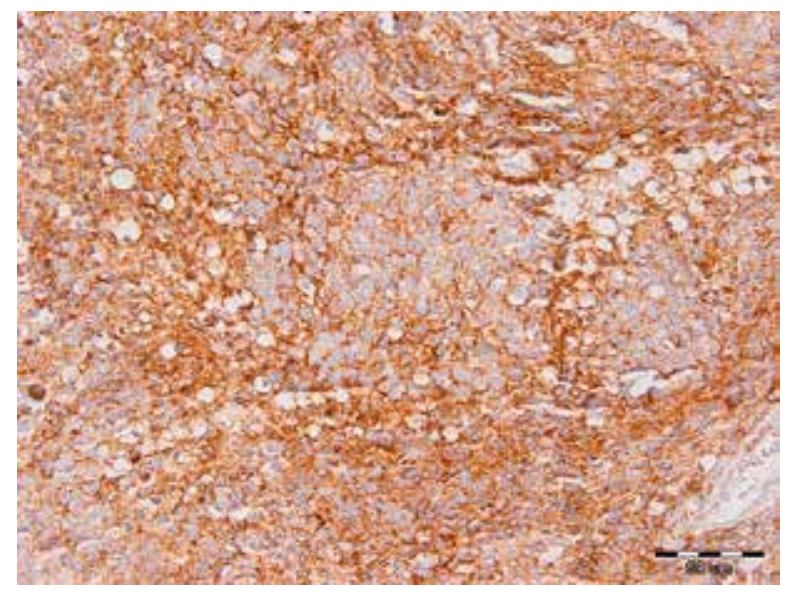

Fig. 3. KAI1/CD82 expression in gliomas graded as Gll. Glioma tissues showed a strong membrane and cytoplasm expression of KAI/1CD82 protein (avidin-biotin staining $\times 200$ ).

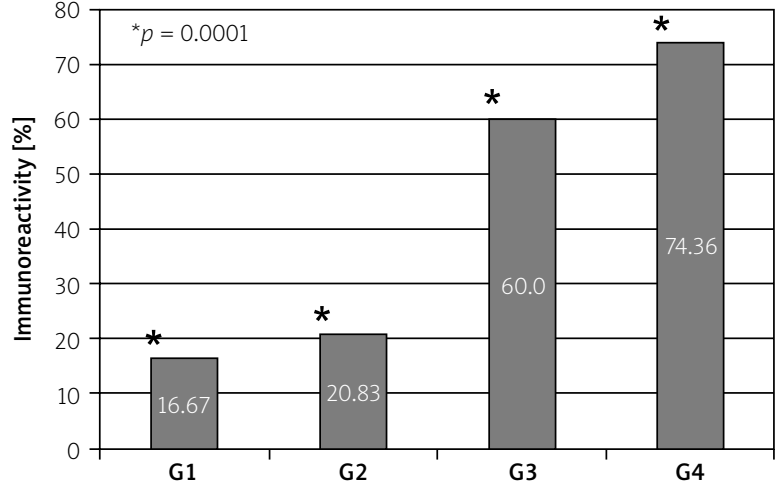

Fig. 2. Significant differences were found for PDGFR $\beta$ expression between gliomas graded as IV/III than in I/II grades $(p=0.0004)$.

for KAI1/CD82 expression between GI/II vs. GIII/IV $(p=0.01)$ (Fig. 4). Similarly, the mean value of KAl1/ CD82 expression in I/II [GI 58.333 \pm 23.1666 (SD), GII $52.916 \pm 32.900$ (SD)] and III/IV [GIII 30.666 28.652 (SD), GIV $25.128 \pm 27.991(\mathrm{SD})]$ tumor grades showed significant differences $(p=0.001)$. Comparing the extent of KAI1 immunoreactivity in relation to tumor grades, a significant downregulation of KAI1/CD82 protein expression in III/IV compared with I/II tumor grades was found ( $p=0.007$ ) (Fig. 5). The increased level of KAI1/CD82 gene expression (3-4-fold) was observed in gliomas with immunoreactivity for KAI1/ CD82 above $50 \%$ of tumor tissue with strong intensity of staining defined as +++.

No correlation between KAI1/CD82 and PDGFR $\beta$ expression was observed in the whole group of gliomas $(p \leq 0.05)$. Moreover, the parallel KAI1/CD82 and PDGFR $\beta$ expression was observed more frequently in a group graded as III and IV than in a group graded

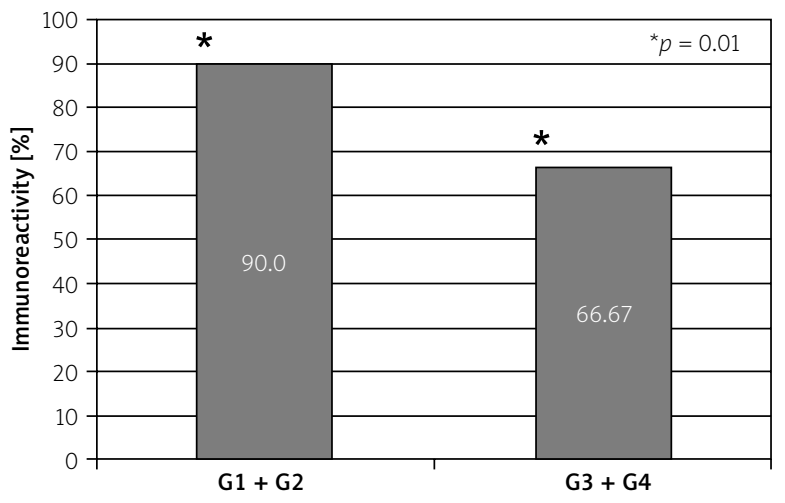

Fig. 4. The mean value of KAI1/CD82 expression in I/II and III/IV glioma grades showed significant differences $(p=0.001)$. 


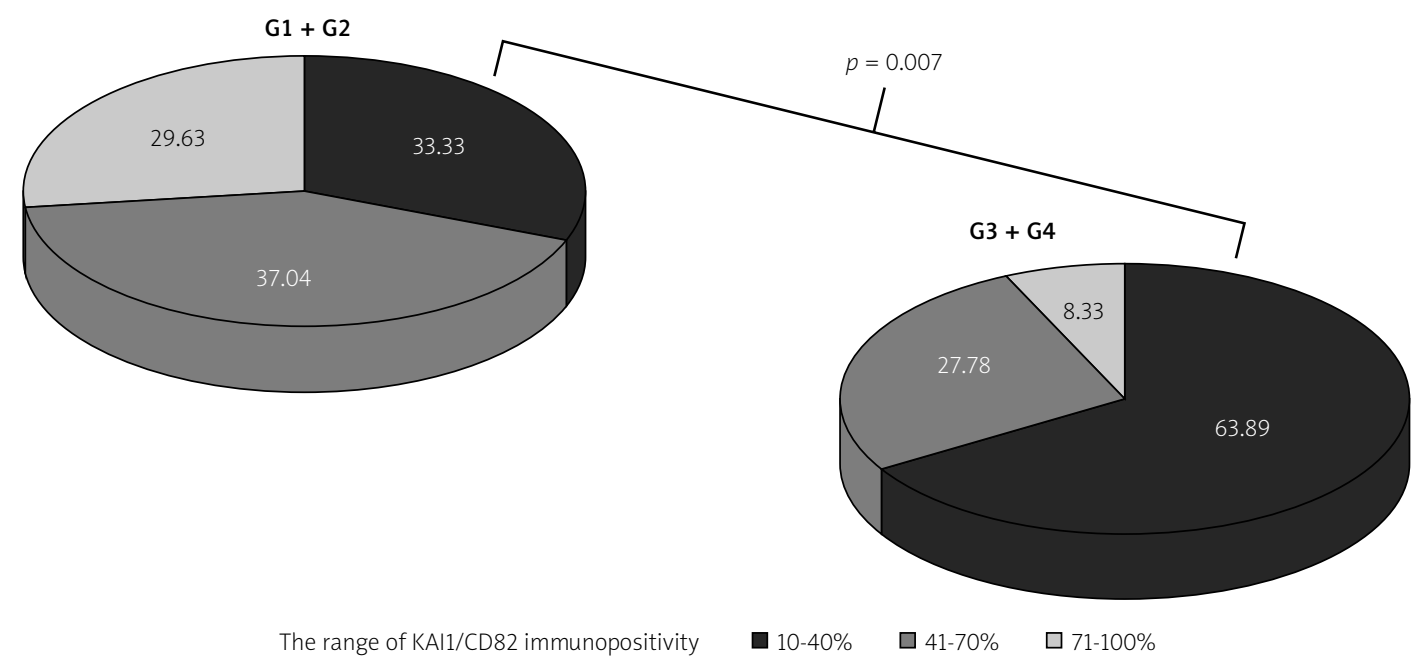

Fig. 5. The extent of KAl1 immunoreactivity related to glioma grades. Significant downregulation of KAl1/ CD82 protein expression was found between gliomas graded as III/IV compared with I/II tumor grades $(p=0.007)$.

as I/II. Observed differences were statistically significant $(p=0.002)$ (Fig. 6).

\section{Discussion}

The common feature of malignant brain tumors is local invasiveness to the surrounding tissue. Specific chemotactic signaling pathways are involved in the regulation of tumor cell motility [22]. In agreement with earlier data $[14,16,21,31]$, we found the PDGFR $\beta$ expression more frequently in grade III and IV than in grade I and II gliomas. Some authors suggest that the autocrine and paracrine stimulation of PDGFR $\beta$ could play an essential role in glial tumorigenesis [4,31]. It was revealed that during glioma progression the PDGFR $\beta$ stimulation can induce the activation of different signaling receptors like EGFR, Notch which lead to dedifferentiation of glioma cells, and increase cell motility and malignancy of the tumor [8,31].

The correlation of PDGFR $\beta$ expression with the WHO high grade gliomas observed in the current study is partly linked to other data which suggest that this receptor is a crucial factor which regulates angiogenesis and might indirectly facilitate dissemination of tumor cells from primary tumor mass and invade normal brain tissue [21]. The role of PDGFR $\beta$ in invasion of tumor cells was revealed by inhibition of cell migration by suppression of PDGFR $\beta$ tyrosine phosphorylation [16]. Based on previous reports and our data we postulate that a high PDGFR $\beta$ expression in low grade gliomas might characterize the subset of gliomas with biological aggressive behavior and suggest that depending on the PDGFR $\beta$ signaling pathway, activation is possible in an early oncogenic event in gliomas [14]. Similarly to other tumors such as breast or liver tumors, a high PDGFR $\beta$ expression in gliomas might contribute to the decreased cell-cell adhesion and promote metastatic capacity of tumor cells [22]. Some data indicate that PDGFR $\beta$ overexpression is associated with epithelial-to-mesenchymal transition (EMT) [22]. Moreover, the PDGFR $\beta$ expression following EMT transient state may induce the metastatic process [27]. Additionally, in this group

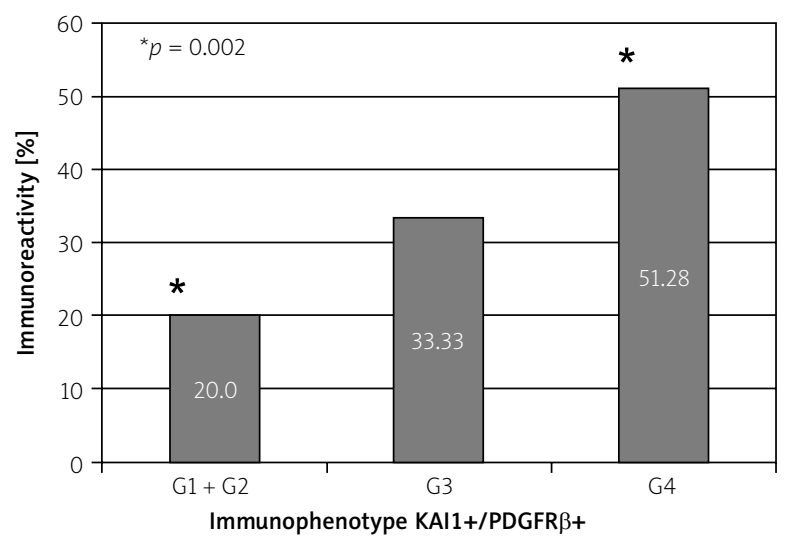

Fig. 6. The parallel KAI1/CD82 and PDGFR $\beta$ expression dominated in gliomas graded as III and IV than in gliomas graded as I/II. Observed differences were statistically significant $(p=0.002)$. 
of tumors a high expression of chitinase-like protein (YKL40) was found [2]. The authors suggested that YKL40 positivity contributes to progression of glioblastoma [2]. In gliomas with a high tumor grade the EMT transient state is often observed and in these tumors both mRNA PDGFR $\beta$ and its protein are highly expressed [18]. Authors claim that glioblastoma cells which have mesenchymal features and possess a high PDGFR $\beta$ expression indicate that this biological feature might characterize the group of tumors with increased cell plasticity [14]. This observation might be compared with our previous study which showed that the E-cadherin expression was weaker or absent in high than low grade gliomas [3]. In the current study, the PDGFR $\beta$ expression was analyzed in the same set of gliomas and obtained data related to the E-cadherin expression (unpublished data).

There are no data describing the role of KAI1/CD82 expression in gliomas by other authors. In the present study we analyzed the mRNA KAI1/CD82 gene and its protein gliomas in order to show the impact of KAI1/CD82 expression on glioma growth. Some authors found that loss of the KAI1/CD82 protein expression is associated with the invasive growth of tumors and metastasis [7,15]. In gliomas the invasive growth is associated with the migration of tumor cells to the surrounding tissue, so the question is whether KAI1/CD82 may suppress the migratory function of tumor cells in gliomas similarly to colorectal carcinoma [27].

The current study found a high KAI1/CD82 expression in low and loss in high grade gliomas. These results are consistent with previous data that KAI1/CD82 expression is associated with increased invasive behavior of solid tumors [7,26,29]. In analyzed glioma specimens overexpression of KAI1/CD82 was observed in glioma cells without malignancy features regardless of the tumor grade whereas the majority of malignant cells were negative for KAI1/CD82 immunostaining. The different pattern of KAI1/CD82 expression observed in this study might reflect the high degree of intratumoral heterogeneity of the primary tumor that progression and metastasis are spawned by selective subclone of cells [28]. Recently, data revealed that upregulation of microRNA-210 induced cell proliferation and migration of glioblastoma cells [32]. We postulate that KAI1/CD82 protein expression in gliomas might play a similar role to that in tumors with metastasis to other organs [7,23]. Firstly, KAI1/CD82 as a metastasis sup- pressor protein might inhibit the migratory ability of glioma cells, so we suggest that a high expression of $\mathrm{KAI} 1 / \mathrm{CD} 82$ in low grade gliomas might reduce the risk of secondary glioblastoma development. Secondly, the other function of KAI1/CD82 protein could be considered in gliomas like regulation by microRNA. An experimental study has shown that KAI1/CD82 is able to inhibit the signaling PI3K/AKT pathway in breast, bladder, and melanoma cancer cell lines $[23,24]$. Taking into account that the PI3K/ AKT pathway is often activated by PDGFR receptors during glioma progression, we suggest that the suppression of the PI3K/AKT pathway by KAI1/CD82 protein might limit the proliferation and spread of glioma cells $[19,23]$.

The present study is the first one to investigate the association between KAI1/CD82 protein and PDGFR $\beta$ expression in gliomas. Interestingly, we found that KAI1/CD82 expression is closely related to PDGFR $\beta$ expression in high grade glioma malignancy. Our results demonstrate that parallel expression of both biomarkers in glioblastomas might identify the cases where KAI1/CD82 might lead to a reduction in PDGFR $\beta$ activity and probably inhibit angiogenesis dependent on PDGFR $\beta$ expression. Our observations are partly consistent with the studies reporting that the KAI1/CD82 attenuated pathway depends of receptor tyrosine kinase (RTKs) activity $[7,24,29]$. Based on earlier reports which describe the association between KAI1/CD82 and surface receptor responsible for a different signaling pathway we suggest that such mechanism may occur in gliomas [31].

\section{Conclusions}

We found that a loss of KAI1/CD82 and an increase in PDGFR $\beta$ expression in gliomas relate to a progressive tumor growth. A correlation between PDGFR $\beta$ and KAI1/CD82 expression in high grade gliomas suggests that a direct or indirect interaction between these proteins might influence the cell motility and invasive behavior of the tumor.

\section{Acknowledgments}

This work was supported by Wroclaw Medical University grant Pbmn 113.

\section{Disclosure}

Authors report no conflict of interest. 


\section{References}

1. Badyopadhyay S, Zhan R, Chaudhuri A, Watabe M, Pai SK, Hirota Sh, Hosobe S, Tsukada T, Miura K, Takano S, Wang Y, Mohinta S, Saito K, Pauza ME, Hayashi S, Wang Y, Mohinta S, Mashimo T, liizumi M, Furuta E, Watabe K. Interaction of KAl1 on tumor cells with DARC on vascular endothelial leads to metastasis suppression. Nat Med 2006; 12: 933-938.

2. Batista KMP, Vega IF, de Eulate-Beramendi SA, Morales JCG, Kurbanov A, Asnel D, Meilan A, Astudillo A. Prognostic significance of the markers IDH1 and YKL40 related to the subventricular zone. Folia Neuropathol 2015; 1: 52-59.

3. Bar JK, Zub L, Lis-Nawara A, Noga L, Jelen M, Paradowski B. Expression and interaction between cell adhesion molecules CD44v6 and E-cadherin in human gliomas. Adv Clin Exp Med 2014; 5: 827-834.

4. Barrett A, Evans IM, Frolov A, Britton G, Pellet-Many C, Yamaji M, Mehta V, Bandopadhyaya R, Ningning L, Brander S, Zachary JC, Frankel P. Critical role for DOK1 in PDGF-BB stimulated glioma cell invasion via p130Cas and Rap1 signalling. I Cell Sci 2014; 127: 2647-2658.

5. Chen H, Shah AS, Reda EG, Grossman SA. Transmission of glioblastoma multiforme after bilateral lung transplantation. J Clin Oncol 2008; 26: 3284-3285.

6. Choi UJ, Jee BK, Lim Y, Lee K-H. KAl1/CD82 decreases Rac1 expression and cell proliferation through PI3K/Akt/mTOR pathway in $\mathrm{H} 1299$ lung carcinoma cells. Cell Biochem Function 2009; 27: 40-47.

7. Dai W, Wang C, Wang F, Wang Y, Shen M, Chen K, Cheng P, Zhang Y, Yang J, Zhu R, Zhang H, Li J, Zheng Y, Lu J, Zhou Y, Xu L, Guo Ch. Anti-miR-197 inhibits migration in HCC cells by targeting KAl 1/CD82. Biochem Bioph Res Commun 2014; 446: 541-548.

8. Dimov I, Tasić-Dimov D, Conic I, Stefanovic V. Glioblastoma multiforme stem cells. Sci World J 2011; 11: 930-958.

9. Dong TJ, Lamb PW, Rinker-Schaeffer CW, Vukanovic J, Ichikawa T, Isaacs JT, Barrett JC. KAl1, a metastasis suppressor gene for prostate cancer on human chromosome 11.p11.2. Science 1995; 268: 884-886.

10. Ellis JA, Canoll P, McCormick PC, Feldstein NA, Anderson RC, Angevine PD, Kaiser MG, McCornik PC, Bruce JN, Ogden AT. Platelet-derived growth factor receptor (PDGFR) expression in primary spinal cord gliomas. J Neurooncol 2012; 106; DOI: 10.1007/s11060-011-0666-6.

11. Malik FA, Sandres AJ, Jiang WG. KAI-1/CD82, the molecule and clinical implication in cancer and cancer metastasis. Histol Histopathol 2009; 24: 519-530.

12. Fertetta M, Uhrbon L, Olofsson T, Pontén F, Westermak B. Sox10 has a broad expression pattern in gliomas and enhances platelet-derived growth factor-B - induced gliomagenesis. Mol Cancer Res 2007; 5: 891-897.

13. Kim ChS, Jung S, Jung TY, Jang WY, Sun HS, Ryu HH. Characterization of invading glioma cells using molecular analysis of leading-edge tissue. J Korean Neurosurg Soc 2011; 50: 157-165.

14. Kim Y, Kim E, Wu Q, Guryanova O, Hitomi M, Lathia JD, Serwanski D, Sloan A, Weil RJ, Lee J, Nlshiyama A, Bao Sh, Hjelmeland AB, Rich JN. Rich platelet-derived growth factor receptors differ- entially inform intertumoral and intratumoral heterogeneity. Genes Development 2012; 16: 1247-1262.

15. Liu X, Guo XZ, Li HY, Chen J, Ren LN, Wu ChY. KAl1 inhibits lymphangiogenesis and lymphatic metastasis of pancreatic cancer in vivo. Hepatobiliary Pancreat Dis Int 2014; 13: 87-92.

16. Liu Y, Le W, Lin Y, Cheang TY, Wang M, Zhang H, Zhang L, Wang S. Gambogic acid induces G0/G1 cell cycle arrest and cell migration inhibition via suppressing PGFR receptor $\beta$ tyrosine phosphorylation and Rac1 activity in rat aortic smouth muscle cells. J Atheroscler Thromb 2010; 17: 901-913.

17. Louis DN, Ohgaki H, Wiestler OD, Cavenee WK. WHO classification of tumours of the central nervous system. $4^{\text {th }}$ ed. International Agency for Research on Cancer (IARC), Lyon 2007; 14-33.

18. Nager M, Bhardwaj D, Canti C, Medina L, Nogues P, Herreros J. $\beta$-catenin signaling in glioblastoma multiforme and gliomainitiating cells. Chem Res Prac 2012; DOI: 10.1155/2012/192362.

19. Nakada M, Kita D, Watanabe T, Hayashi Y, Teng L, Pyko IV, Hamada Jl. Aberrant signaling pathways in glioma. Cancer 2011; 3: 3242-3278.

20. Nazarenko I, Hede SM, He X, Hedren A, Thompson J, Lindstrom MS, Nister M. PDGF and PDGR receptors in glioma. Upsala J Med Sci 2012; 117: 99-112.

21. Pfister C, Pfrommer H, Tatagiha MS, Roser F. Vascular endothelial growth factor signals through platelet-derived growth factor receptor $\beta$ in meningiomas in vitro. Br J Cancer 2012; 107: 1702-1713.

22. Steller EJA, Raats DA, Koster J, Rutten B, Govaert KM, Emmik BL, Snoeren N, van Hooff SR, Holstege F, Maas C, Rinkes HM, Kranenburg O. PDGFRB promotes liver metastasis formation of mesenchymal-like colorectal tumor cells. Neoplasia 2013; 15: 204-217.

23. Tang T, Cheng Y, Martinka M, Ong CH, Li G. Prognostic significance of KAl1/CD82 in human melanoma and its role in cell migration and invasion through the regulation of ING4. Carcinogenesis 2014; 1: 86-95.

24. Tsai $Y C H$, Weissman AM. Dissection the diverse function of the metastasis suppressor CD82/KAl1. FEBS Lett 2011; 585: 3166-3173.

25. Wan L, Pantel K, Kang Y. Tumor metastasis: moving new biological insights into the clinic. Nature Med 2013; 11: 1450-1464.

26. Wang G, Jiang H, Xu H, Sun Q, Zhou Y, Xiang P, Cheng Z, Zhang Y, Zhou Y, Guo Q, Du X, Xu S, Ma S, Chen Z. Clinical significance of KAI1/CD82 protein expression in nasopharyngeal carcinoma. Oncol Lett 2015; 9: 1681-1686.

27. Wu Q, Yang Y, Wu SH, Li W, Zhang NA, Dong X, Ou Y. Evaluation of the correlation of KAI/CD82, Cd44, MMP7, $\beta$-catenin in the prediction of prognosis and metastasis in colorectal carcinoma. Dig Pathol 2015; 10: 176-182.

28. Wu X, Northcott PA, Dubuc A. Clonal selection drives genetic divergence of metastatic medulloblastoma. Nature 2012; 482: 529-533.

29. You JJ, Hon B, Madigan MC, Rowe A, Sajinovic M, Russell PJ, Jackson P. An inverse relationship between KAl1 expression, invasive ability, and MMP-2 expression and activity in bladder cancer cell lines. Urol Oncol 2012; 30: 502-508. 
30. Yu L, Zhou L, Wu Sh, Gong X, Feng Zh, Ma L, Zhu B, Yao N, Wang D, Dong H. Clinicopathological significance of cancer stem cells marked by CD133 and KAl1/CD82 expression in laryngeal squamous cell carcinoma. World J Surg Oncol 2014; 12: 118.

31. Zhang J, Chen T, Mao Q, Lin J, Jia J, Li Sh, Xiong W, Lin Y, Liu Zh, Liu X, Zhao H, Wang G, Zheng D, Qiu Sh, Ge J. PDGFR- $\beta$-activated ACK1-AKT signaling promotes glioma tumorigenesis. Int J Cancer 2015; 136: 1769-1780.

32. Zhang Sh, Lai N, Liao K, Sun J, Lin Y. MicroRNA-210 regulates cells proliferation and apoptosis by targeting regulator of differentiation 1 in glioblastoma cells. Folia Neuropathol 2015; 3: 236-244. 